\title{
Detecting Change in Snapshot Sequences
}

\author{
Mingzheng Shi and Stephan Winter \\ Department of Geomatics, University of Melbourne, Victoria, 3010, Australia \\ m.shi@pgrad.unimelb.edu.au, winter@unimelb.edu.au
}

\begin{abstract}
Wireless sensor networks are deployed to monitor dynamic geographic phenomena, or objects, over space and time. This paper presents a new spatiotemporal data model for dynamic areal objects in sensor networks. Our model supports for the first time the analysis of change in sequences of snapshots that are captured by different granularity of observations, and our model allows both incremental and nonincremental changes. This paper focuses on detecting qualitative spatial changes, such as merge and split of areal objects. A decentralized algorithm is developed, such that spatial changes can be efficiently detected by in-network aggregation of decentralized datasets.
\end{abstract}

\section{Introduction}

Many spatial phenomena are continuously changing in time and space [5]. Environmental monitoring, for example, focuses especially on areas of environmental disturbance, such as grazing, fertilizing, pollution, and logging. Environmental disturbance is one of the key driving forces for environmental changes, and different disturbance events can result in diverse changes. For example, a logging event can result in the decrease of forest coverage and the change from forest to heath land. A flooding event can result in the increase of soil moisture and the transform from heath land to wetland [9].

Wireless sensor networks (WSNs) are increasingly being used in spatial applications to detect change in the environments. A WSN is a network of computing devices that can collaborate via radio communications. A WSN is also a network of observation devices, since each node in the WSN is equipped with sensors that enable thematically fine-grained observation of the environments. A WSN is able to observe change in real-time, and hence, with almost any temporal granularity (but typically coarser spatial granularity).

In the context of geographic information science, snapshot-based and eventbased approaches have been used to model wireless sensor networks. Snapshotbased approach are commonly used in applications, where sensor nodes are tasked to periodically sense and transmit snapshots of an environment by setting the WSN to a certain temporal sensing resolution (e.g., [16]). Although the snapshot approach is more practical, theoretical studies (e.g., [18]) are more interested in event-based model of WSNs due to its advantages of detecting salient changes or events. 


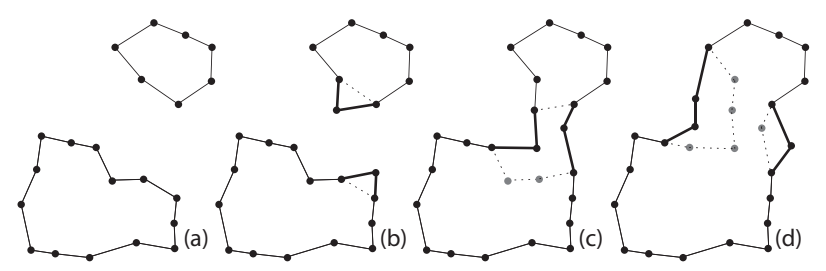

Fig. 1. The evolving of spatial objects over time. (a)-(d) are at different time steps, e.g., $t_{1}, t_{2}, t_{3}$, and $t_{4}$.

However, practically also event-based models are subject to granularity effects. By certain spatial and temporal resolution of observation, events may not be recorded somewhere, sometime. Even if someone may argue that continuous observation is possible with future technologies, there is still the problem of redundancy of observations. Large amount of energy can be consumed to observe an environment where no change has occurred. In this circumstance, this paper develops a new spatiotemporal data model that incorporates both snapshotbased and event-based approaches, such that the model can adapt to different granularities of observations, and can detect and analyze spatial changes.

In our spatiotemporal data model, a WSN is modeled as a set of point objects with point observations, and the primary structure in our model are edges. Edges are important components for spatial objects. For example, an area is embedded in a polygon that is constituted as a sequence of edges. By sensor network point observations, geographic phenomena will be abstracted as areal objects that consist of points, and these points will then be organized as sequence of edges. Since temporal granularity of observation is allowed in our model, geographic phenomena are represented as sequences of snapshots. Our model supports the analysis of change in sequences of snapshots that is captured by different granularity of observations. Figure 1 illustrates an example about the representation of dynamic areal objects in a sensor network.

This paper has four major contributions. Firstly, we propose a new data structure that ensures the boundaries of areal objects are always closed traversable trails. Secondly, we propose deleted and inserted edges to represent changes of areal objects in snapshot sequences. The sequences of deleted and inserted edges always form closed traversable trails, and there are eight different types of these closed trails that can be used to distinguish six topological changes and two non-topological changes. Thirdly, our approach can support both incremental and non-incremental changes. And finally, we develop a decentralized algorithm to efficiently detect the eight different types of changes in a sensor network.

\section{Related Work}

The change investigated in this paper is the change of spatial entities. A change occurs whenever spatial entities possess different spatial attributes at different 
times [2]. Grenon and Smith [4] classify entities in the spatiotemporal world into two categories: one is continuants, and the other is occurrents. The study of this paper is on the perspective of continuants that exist at a given time at a given level of granularity and undergo different types of changes over time. Hornsby and Egenhofer [5] suggest a qualitative representation of change. Their notion of change is based on object identity, and a set of operations that either preserve or change identity. This paper applies their concept of identity assuming that sensor network can be set up with a certain appropriate granularity of observations for a certain environmental phenomenon, according to the sampling theorem [14].

Grenon and Smith [4] propose SNAP and SPAN ontologies. The SNAP ontology has a snapshot-based view, where entities are organized as temporal sequences of snapshots. On the other hand, event-based spatiotemporal data models in SPAN ontology (e.g., $[10,11,17])$ can be understood as a chronicle-based model that is dual to the snapshot-based model [3]. Snapshot-based and eventbased approaches are commonly used in wireless sensor network applications.

Lian et al. [6] propose a gradient boundary detection approach, where snapshots of a monitored region are transmitted to a designated central computer via the sink of the network at certain time intervals. A snapshot at a given time $t$ is abstracted as a contour map, which consists of gradient boundaries. Only the nodes on the gradient boundaries need to report to the sink, which constructs a contour map. A unique work for collecting snapshot data is proposed by Skraba et al. [15]. Their method is called a sweep, i.e., a wavefront that traverses the whole network and passes all nodes in the network exactly once. These approaches are limited to static snapshots. They cannot dynamically analyze snapshot sequences to derive salient changes.

Duckham et al. [1] develops a model to track salient changes or events in the environment. They model a sensor network as a triangulation network. The triangulation can change over time in response to the movement of spatial phenomena. Worboys and Duckham [18] also use triangulations, and spatial objects are represented as a set of triangles. Changes of spatial objects are represented as the insertion or deletion of triangles from the set. However, their approach is limited to incremental changes, i.e., the change of a single triangle at each time step. Sadeq and Duckham [13] investigate different sensor network structures for detecting topological changes. Several commonly-used neighborhood structures, such as Delaunay triangulation, Gabriel graph, relative neighborhood graph, and greedy triangulation, have been tested. Their approach also assumes incremental changes. Their classification of spatial changes is based on the theoretical study of Jiang and Worboys [8].

A large amount of existing work on qualitative approaches to characterize topology and topological changes in sensor network exists, e.g., [7]. This paper is distinguished from other related work by using edge sequences for qualitative reasoning. We use deleted and inserted edges to represent changes of areal objects, and the sequences of deleted and inserted edges are always closed trails regardless the number of nodes on the trails. Thus, our approach allows for the first time both incremental and non-incremental changes. 


\section{Change Representation in Sensor Network}

A sensor network can be modeled as a directed planar graph $G=(V, E)$, which is built by Delaunay triangulation [12]. In this paper we assume that the sensor network is dense enough to ensure the construction of a triangulation framework. In the graph $G, V$ is a set of nodes and $E$ is a set of edges, e.g., $\left(v, v^{\prime}\right)$, which represent the direct communication links between the nodes $v, v^{\prime} \in V$. We assume that $E$ is symmetric, i.e., if $\left(v, v^{\prime}\right) \in E$, then $\left(v^{\prime}, v\right) \in E$. Note that the direction of a directed edge will be illustrated in a figure when it is relevant, otherwise the representation of $G$ can be simplified as in Figure 2(a).

The set of neighbors of $v \in V$ is denoted as $n b r(v)=\left\{v^{\prime}:\left(v, v^{\prime}\right) \in E\right\}$. For example in Figure 2(a), $n b r(v)=\{a, b, c, d, e\}$, and the set $n b r(v)$ is sorted into clockwise order. Each node only stores data about itself and its immediate neighbors.

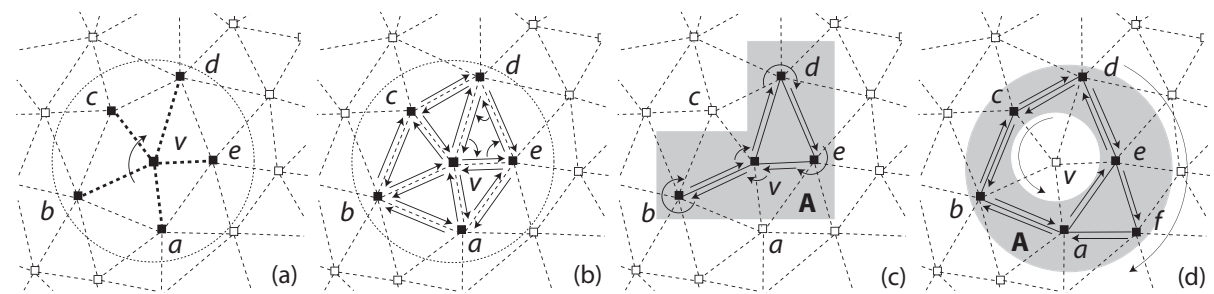

Fig. 2. Sensor network structure. (a) The set $\operatorname{nbr}(v)$ of each node $v$ is sorted into clockwise order. (b) The directed edges and cycles of a node $v$. (c) The traversal of boundary edges based on the clockwise rule. (d) A region with a hole can be detected by the traversal orientations.

The set of directed edges adjacent to a node $v$ is denoted as edge $(v)$. Since $E$ is symmetric, the set edge $(v)$ can be derived from $n b r(v)$, i.e., edge $(v)=$ $\left\{\left(v, v^{\prime}\right): v^{\prime} \in n b r(v)\right\} \cup\left\{\left(v^{\prime}, v\right): v^{\prime} \in n b r(v)\right\}$. In Figure 2(b), for example, the node $v$ has five neighbors, so that $v$ has ten directed edges. The set edge $(v)$ is also sorted in clockwise order, and for a same neighboring node $v^{\prime}$, the edge $\left(v^{\prime}, v\right)$ is always the next edge of the edge $\left(v, v^{\prime}\right)$, as in Figure 2(b).

A node $v$ and its neighbors are organized into directed cycles, or simply cycles. A cycle is denoted as $(v, b, a, v)$, where the three nodes $v, a$ and $b$ are distinct, and there is an edge for any two consecutive nodes in the cycle, i.e., $(v, b),(b, a)$, $(a, v) \in E$. Since $n b r(v)$ is in clockwise order, all the cycles are counterclockwise, and an example, i.e., $(v, e, d, v)$, is shown in Figure 2(b). Based on the above definition, we can define the representation of areal objects in a sensor network:

Definitions: A directed edge $\left(v, v^{\prime}\right) \in E$ is an object edge, if both $v$ and $v^{\prime}$ are located in an areal object. A cycle $(v, b, a, v)$ is an object cycle, if all the vertexes of the cycle, i.e., $v, a, b$, are in an areal object. An object edge $\left(v, v^{\prime}\right) \in E$ 

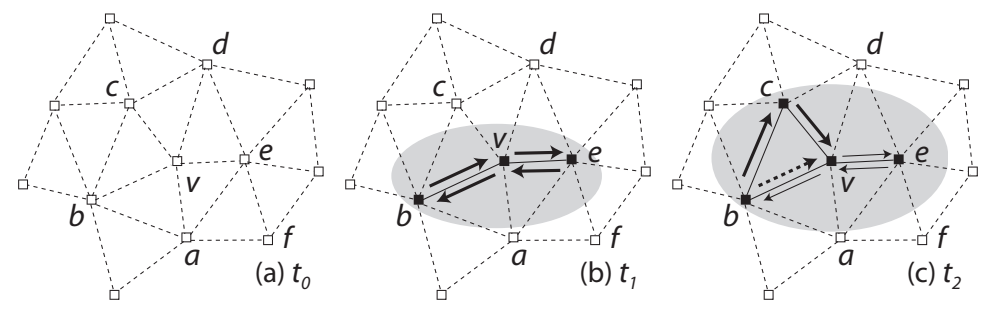

Fig. 3. Inserted and deleted boundary edges are marked by thick solid-line arrows and thick dashed-line arrows respectively. Only boundary edges are illustrated in the figure.

is a non-boundary edge, if the edge belongs to an object cycle. An object edge $\left(v, v^{\prime}\right) \in E$ is a boundary edge, if it does not belong to any object cycle.

Areal objects can be represented as sequence of boundary edges. An example is shown in Figure 2(c), in which the nodes $b, d, e$ and $v$ are located in an areal object $A$. The edges $(b, v),(v, d),(d, e),(e, v),(v, b)$ are boundary edges, since they do not belong to any object cycles. Usually only boundary edges will be illustrated in the figures, as in Figure 2 (c) and (d).

Traversals on boundary edges of areal objects always form closed trails. For example, in Figure 2(c), suppose the traversal is started at node $b$, then the traversal will be a closed trail followed the clockwise rule: $b \rightarrow v \rightarrow d \rightarrow e \rightarrow$ $v \rightarrow b$. Formally, the path of a set of connected boundary edges is a Eulerian trail, since the number of boundary edges in each node is always even.

An important property of these trails is the traversal orientation. Given a region with a hole, for example in Figure 2(d), external traversals of the region are clockwise and internal traversals of the hole are counterclockwise. Since the area of a polygon is positive if the vertexes of the polygon are arranged in a counterclockwise order, and negative if they are in clockwise order, the result of area calculation can be used to determine the ordering of the vertices of a polygon, and thus the orientation of a traversal.

In our model, the change of areal objects will be captured as sequences of snapshots in the sensor network. In each snapshot, sequences of boundary edges are used to represent areal objects. Since areal objects are evolving over time, the sequences of boundary edges would dynamically change at different snapshots, as in Figure 3(a)-(c). The change of boundary edges between two consecutive snapshots is represented by the insertion and deletion of boundary edges:

Definitions: A boundary edge $\left(v, v^{\prime}\right) \in E$ is an inserted boundary edge, or simply an inserted edge, at time $t_{i}$ if the edge is not a boundary edge at previous time $t_{i-1}$, but become a boundary edge at time $t_{i}$. A boundary edge $\left(v, v^{\prime}\right) \in E$ is a deleted edge at time $t_{i}$ if the edge is a boundary edge at previous time $t_{i-1}$, but is not a boundary edge at time $t_{i}$.

We assume the sensor reading is binary, i.e., $\{0,1\}$, where the reading of 1 represents that the sensor node is located in an areal object. We define that if a node $v$ changes its sensor readings, e.g., from 1 to 0 , at time step $t_{i}$, then $v$ 


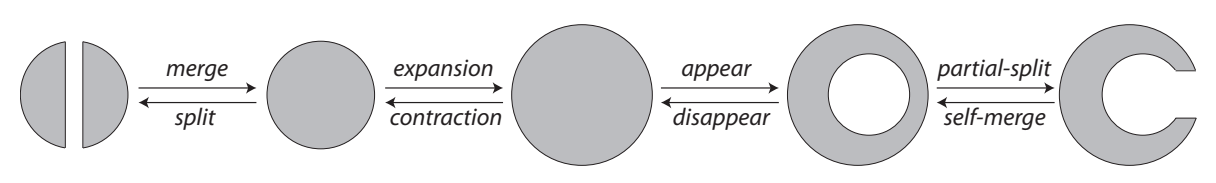

Fig. 4. Six topological changes and two non-topological changes.

is an active node at $t_{i}$. For example, in Figure 3, nodes $b, e$, and $v$ are active nodes at $t_{1}$, and node $c$ is the only active node at $t_{2}$. We define that between two consecutive time steps $t_{i}$ and $t_{i-1}$, a change of areal object is incremental if there is one and only one active node at time step $t_{i}$. In Figure 3, the change between $t_{0}$ and $t_{1}$ is non-incremental and the change between $t_{1}$ and $t_{2}$ is incremental. Our model supports both incremental and non-incremental changes.

\section{Detecting Change}

The traversals on deleted and/or inserted edges can be used to distinguish eight different types of changes of areal objects. These eight types of changes include six topological changes and two non-topological changes. The six topological changes are appearance, disappearance, merge, split, self-merge and partial-split $[8,13]$. The two non-topological changes are expansion and contraction. Examples of the eight types of changes are shown in Figure 4.

As will be discussed, different types of changes will have different types of traversals on deleted/inserted edges, and all of the traversals will form closed traversable trails. We discuss the eight types of changes in four groups.

\subsection{Appearance and Disappearance}

There is a closed traversable trail for the appearance of areal objects. In Figure $5(\mathrm{a})$, an areal object appears, and a set of boundary edges has been inserted. The set of inserted boundary edges forms a closed trail. Suppose the traversal starts at node $i$, then the trail is: $i \rightarrow a \rightarrow b \rightarrow c \rightarrow d \rightarrow e \rightarrow f \rightarrow g \rightarrow h \rightarrow i$. An abstract representation of the traversal in Figure 5(a) is shown in (b), where the solid-line arrow represents a traversal on inserted edges, and the circle represents a node that has a node identity of $i$. Note that Figure 5 illustrates the stage when changes have occured but areal object identities have not yet been updated.

In Figure 5(c), an areal object disappears, and all the boundary edges with areal object identity of $A$ have been deleted. And in Figure 5(d), the dashed-line arrow denotes a traversal on deleted edges. Note that areal object identity is decentrally stored in each boundary edge of the areal object.

\subsection{Expansion and Contraction}

For the expansion and contraction of areal objects, the closed trail will include two segments. One segment of the traversal consists of inserted edges, 
and another segment consists of deleted edges. In Figure 5(e), for example, an areal object expands. The inserted edges form one segment of the traversal: $b \rightarrow c \rightarrow d \rightarrow e \rightarrow f \rightarrow g \rightarrow h$. The deleted edges form another segment: $h \rightarrow j \rightarrow b$. Notice that the second segment of the traversal is in reverse direction. Figure 5(f) illustrates that the two segments of the traversal are connected by two nodes $b$ and $h$. The two nodes $b$ and $h$ are called the transition nodes of the traversal. Segment 2 is aware the areal object identity $A$, while Segment 1 do not have areal object identity yet.

Similarly, in Figure 5(g) and (h), an areal object contracts, and there are two segments that is connected by two transition nodes $b$ and $h$. Section 5 will demonstrate how expansion and contraction can be distinguished: the traversal of expansion consists of (1) inserted edges and (2) deleted edges, and the traversal of contraction consists of (1) deleted edges and (2) inserted edges.

\subsection{Merge and Split}

The closed trail for the merge of two areal objects includes four segments. Figure 5 (i) shows a merge of two areal objects $A$ and $B$. The first segment of the traversal is on inserted edges from node $a$ to node $d: a \rightarrow b \rightarrow c \rightarrow d$. Two transition nodes $a$ and $d$ are located in different areal objects, i.e., $A$ and $B$ respectively. The second segment of the traversal is on the deleted edges of areal object $B$ from node $d$ to node $m: d \rightarrow e \rightarrow m$, and the deleted edges have the areal object identity $B$, as shown in Figure $5(\mathrm{i})$ and (j). The third segment will start from areal object $B$ and return to areal object $A$ via inserted edges: $m \rightarrow f \rightarrow g$. Finally, the fourth segment of the traversal will return to the original node $a$ via the deleted edges of areal object $A: g \rightarrow j \rightarrow a$. Note that Segments 2 and 4 are in reverse direction. These four segments of traversal are connected by four transition nodes $a, d, m$, and $g$, in which two nodes are located in areal object $A$, and the other two are in areal object $B$.

The traversal of a split also includes four segments, and the four segments are in the sequences of (1) deleted edges and (2) inserted edges and (3) deleted edges and (4) inserted edges, as in Figure 5(k) and (l). The four segments of traversal are also connected by four transition nodes $a, d, m$, and $g$. Segments 1 and 3 on deleted edges are aware the areal object identity $A$. In comparison, the four traversal segments of a merge are in a different order: (1) inserted edges and (2) deleted edges and (3) inserted edges and (4) deleted edges, as in Figure $5(\mathrm{j})$. Both of the traversals in Figure 5(j) and (l) start at node $a$. The selection of the traversal starting node will be discussed in Section 5 .

\subsection{Self-merge and Partial-split}

In Figure 5(m), an areal object $A$ self-merges into a region with a hole. The traversal of self-merge has a similar structure as a merge: (1) inserted edges and (2) deleted edges and (3) inserted edges and (4) deleted edges, as shown in Figure 5(n). Segments 2 and 4 of a self-merge have the same areal object identity $A$, as illustrated in Figure 5(m) and (n). In comparison, Segment 2 and 

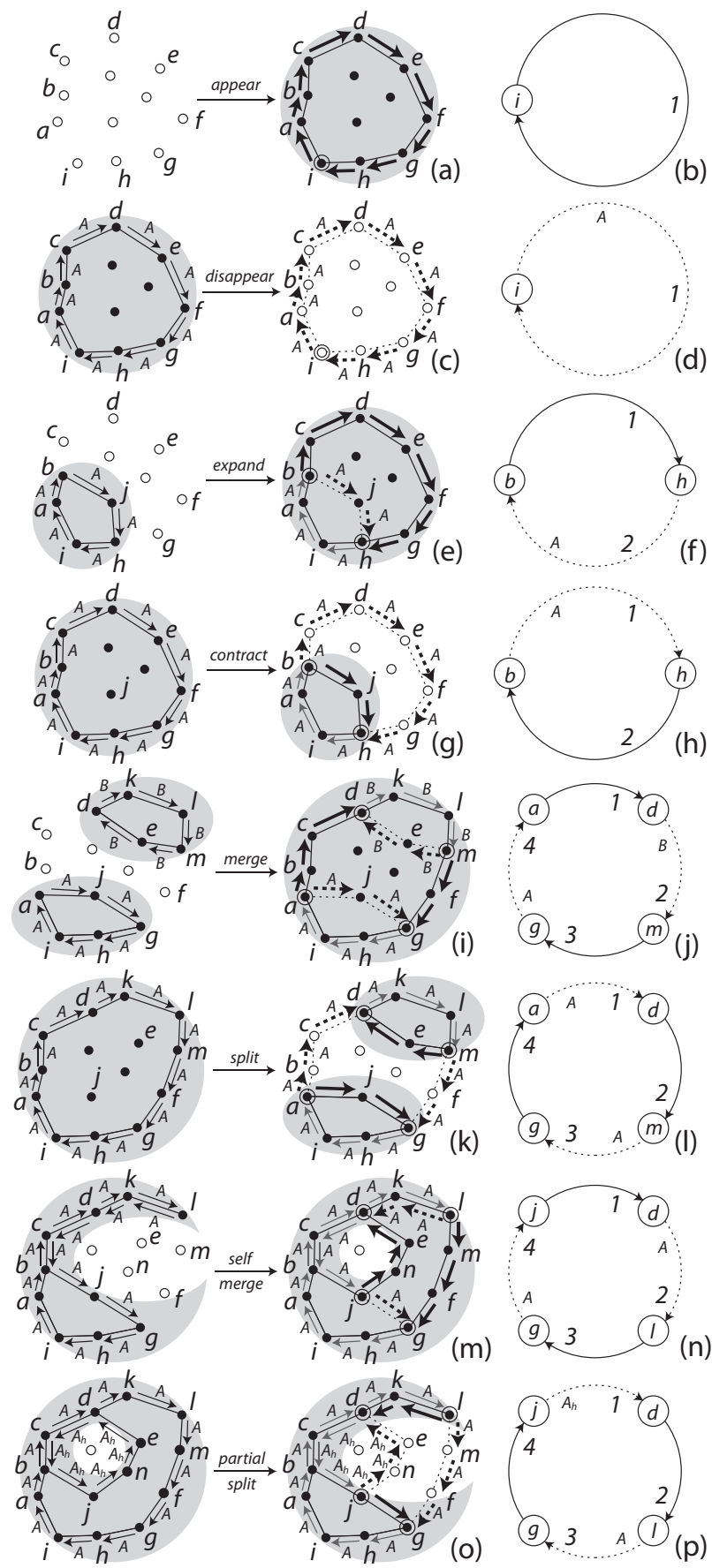

Fig. 5. Eight traversals form eight different closed traversable trails 


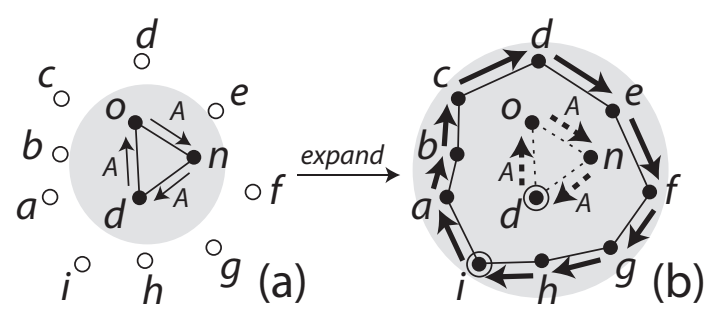

Fig. 6. Other types of traversals can be considered as the combinations of the eight basic traversal types. The traversal in (b) is a combination of two traversals in Figure $5(\mathrm{~b})$ and (d).

4 of a merge in Figure 5(i) and (j) have different areal object identities, i.e., $B$ and $A$ respectively. Thus, merges and self-merges can be distinguished by the areal object identities of the traversal segments, as in Figure 5(j) and (n).

In Figure 5(o), an areal object with a hole partial-splits. The areal object with a hole consists of two close trails: one is internal for the hole and the other is external for the areal object, as already introduced in Section 3. The areal object identity for a hole of an areal object $A$ is specified as $A_{h}$. Figure 5(o) shows that all the internal edges with $A_{h}$ form a closed trail: $b \rightarrow j \rightarrow n \rightarrow e \rightarrow d \rightarrow c \rightarrow b$. Similar to splits, the traversal for partial-split has four segments, and the order is (1) deleted edges and (2) inserted edges and (3) deleted edges and (4) inserted edges. For a split, Segments 1 and 3, in Figure 5(1), have the same areal object identity $A$, while in the case of partial-split in Figure 5(p), Segment 1 has an areal object identity of $A_{h}$ and Segment 3 has a different identity $A$.

\subsection{Summary}

As a summary, there are eight different types of basic traversals on deleted and inserted edges. These eight traversals can be distinguished based on their segments. Each type of traversal can uniquely identify one type of change. There are possible other types of traversals, and they can be considered as the combinations of the eight basic traversal types. For example, in Figure 6, an areal object expands, and the deleted and inserted edges have been separated into two closed trails: i.e., $d \rightarrow o \rightarrow n \rightarrow d$ and $i \rightarrow a \rightarrow b \rightarrow c \rightarrow d \rightarrow e \rightarrow f \rightarrow g \rightarrow h \rightarrow i$. The traversals in Figure 6 can be considered as a combination of the two traversals in Figure 5(b) and (d). Our algorithms are able to handle the combination of the eight basic traversals such that different change types can be identified.

\section{Algorithm}

As discussed in Section 4, there are eight different types of traversals that can be used to distinguish eight types of changes. This section will provide decentralized 
algorithms for traversal organization and change detection. The basic idea of our algorithm is to initialize a traversal at a given node $v$ with a message, and the message will be passed from one node to another during the traversal. Also, decentralized data will be aggregated into the message during the traversal. Since all the traversals form closed trails, the aggregated message will return to its origin node $v$, so that node $v$ will be able to detect different types of changes based on the aggregated message.

\subsection{Messages}

Since areal object identities of traversal segments can be used to identity different types of changes as in Figure 5, relevant areal object identities will be aggregated during the traversal. A message will be initialized at the beginning of a traversal for data aggregation in the network. A message initialized at a node $v$ is denoted as: $\operatorname{msg}(v)=\left(p_{1}, p_{2}, p_{3}, p_{4}\right)$.

The four elements, i.e, $p_{1}, p_{2}, p_{3}$, and $p_{4}$, denote the areal object identities of the four segments in a traversal. We use $m s g(v) . p_{1}$, for example, to represent the element $p_{1}$ in $m s g(v)$. In the beginning of a traversal, all the elements in a message are empty, and the message can be represented as: $m s g(v)=(\emptyset, \emptyset, \emptyset, \emptyset)$.

The transition nodes are responsible to update the elements of a message during a traversal. For example, the closed trail for a split in Figure $5(\mathrm{k})$ consists of four transition nodes $a, d, m$, and $g$, and four segments. If node $a$ initializes a traversal with a message $m s g(a)$, then node $a$ will update the element $m s g(a) \cdot p_{1}$, and $d, m$, and $g$ will update $m s g(a) \cdot p_{2}, m s g(a) \cdot p_{3}$, and $m s g(a) \cdot p_{4}$ respectively. Finally, the traversal will return to the initial node $a$ with a message that contains all the information about the closed trail.

All the edges in the same segment have the same areal object identity. For example, in Figure $5(\mathrm{k})$, the edges $(a, b),(b, c)$ and $(c, d)$ in the first segment have the same identity of $A$, and thus $m s g(a) \cdot p_{1}=A$. Similarly, $m s g(a) \cdot p_{2}=I$, $m s g(a) \cdot p_{3}=A$, and $m s g(a) \cdot p_{4}=I$. Note that $m s g(a) \cdot p_{2}=I$ and $m s g(a) \cdot p_{4}=I$ are used to specify that the second and fourth segments of the traversal are on inserted edges and areal object identities have not been provided. In Figure $5(\mathrm{k})$, a completed message for the traversal started at node $a$ would be: $m s g(a)$ $=(A, I, A, I)$.

\subsection{Absent Traversal Segments}

In some traversals, it is possible that there is no edge in a segment, and the segment is regarded as an absent segment. For example, in Figure 7(b), if node $b$ starts a traversal, then the traversal would only contains three segments: $b \rightarrow$ $c \rightarrow d$ on inserted edges, $d \rightarrow c \rightarrow a$ on inserted edges, and $a \rightarrow b$ on a deleted edge. Since a traversal of merge has (1) inserted edges and (2) deleted edges and (3) inserted edges and (4) deleted edges, as in Section 4.3, we regard the second segment on deleted edges as an absent segment at node $d$. The areal object identity for the absent segment should be provided by node $d$. As shown in Figure 7 (b), node $d$ can acquire the areal object identity, i.e., $B$, from its boundary edges 

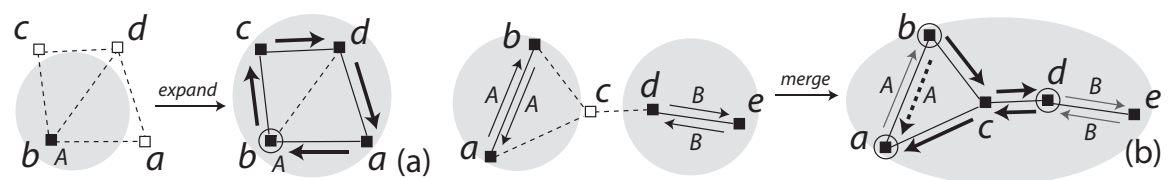

Fig. 7. It is possible that there are absent segments in a traversal. Transition nodes are marked by circles. (a) A segment on deleted edges is absent at node $b$. (b) A segment on deleted edges is absent at node $d$.

$\{(d, e),(e, d)\}$. The completed message initialized by node $b$ would be: $m s g(b)=$ $(I, B, I, A)$.

Generally, in traversals of merge, split, self-merge and partial-split, the first and third segments always exist, while the second and fourth segments may be absent. In the traversals for expansion and contraction, the first segment always exists, while the second segment may be absent, as an example in Figure 7(a).

\subsection{Transition Edges}

Since our algorithm should be able to efficiently organize traversals for decentralized data aggregation, only a small subset of sensor nodes should be nominated for initializing traversals. We define that an inserted or deleted edge is a transition edge if the edge connects an active node and a non-active node.

For example, in Figure $5(\mathrm{k})$, the deleted edge $(a, b)$ is a transition edge that has a initial non-active node $a$ and a terminal active node $b$. Also $(c, d)$ is a transition edge, but the edge begins at an active node $c$ and ends at a non-active node $d$.

Transition edges are always in pairs. If there is a transition edge with an initial non-active node and a terminal active node, then there always exists another transition edge with an initial active node and a terminal non-active node. A pair of transition edges is always connected by a trial. For example, in Figure $5(\mathrm{k}),(a, b)$ and $(c, d)$ are connected by the trial $a \rightarrow b \rightarrow c \rightarrow d$.

If a node $v$ is a non-active node and is the initial node of a transition edge, then node $v$ has the responsibility to initialize a traversal (type 1 ) with a message $m s g(v)$. For example, in Figure $5(\mathrm{k})$, node $a$ is a non-active node, and it is the initial node of a transition edge $(a, b)$, so $a$ should start a traversal with a new message $m s g(a)$. The traversal will visit nodes $b, c, d, e, m, f, g$, and $j$, and return to the starting node $a$ with a completed message $m s g(a)$. Similarly, in Figure $5(\mathrm{k})$, the non-active node $m$ is the initial node of $(m, f)$, and thus node $m$ should also initialize a traversal.

In the case of appearance or disappearance of an areal object, all the nodes in the areal object are active nodes, and thus there is no transition edge. Another type of traversal (type 2) is required for these cases. Although our algorithm includes the organization and combination of traversals type 1 and type 2, the details are not further discussed. 


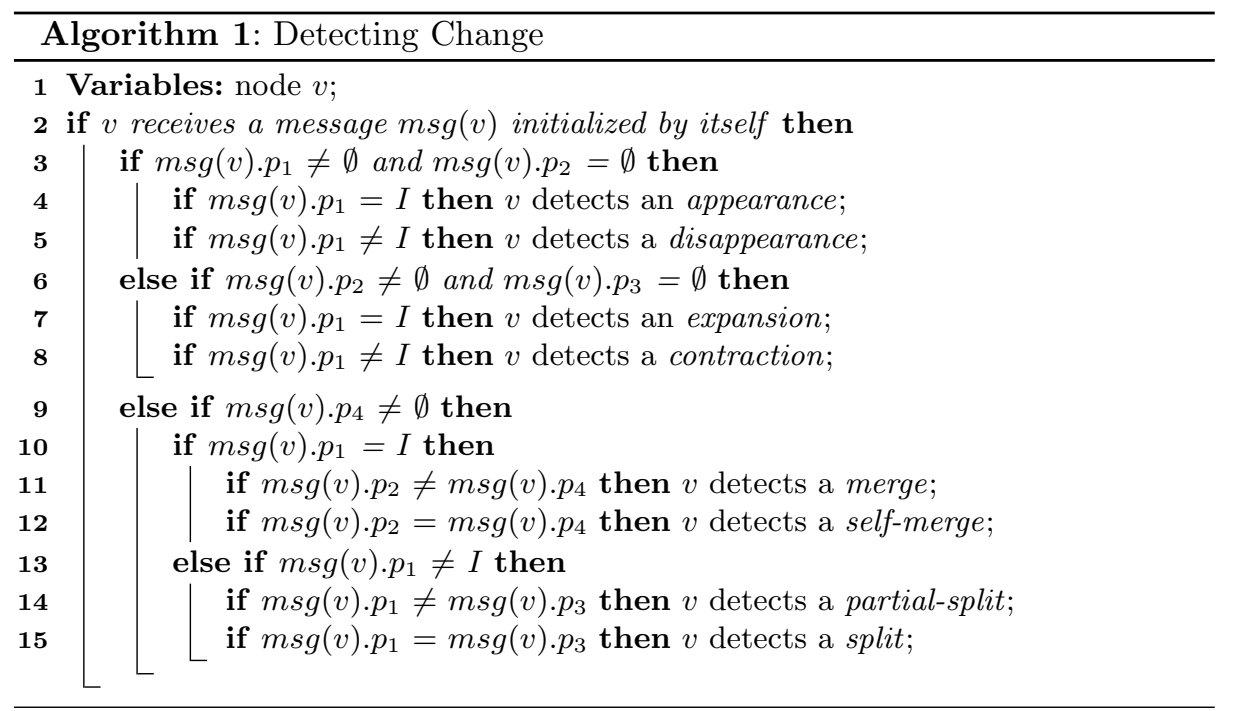

\subsection{Detecting Change}

If a node $v$ receives a message $m s g(v)$ initialized by itself, then node $v$ is able to detect different types of changes based on the message $m s g(v)$ (see Algorithm 1 , line 2). If the message contains only one segment, i.e., $m s g(v) \cdot p_{1} \neq \emptyset$ and $m s g(v) \cdot p_{2}=\emptyset$, then node $v$ detects an appearance or a disappearance (lines $3-5)$. If the segment is on inserted edges, i.e., $m s g(v) \cdot p_{1}=I$, then node $v$ detects an appearance (line 4$)$. If the segment is on deleted edges, i.e., $m s g(v) \cdot p_{1} \neq I$, then node $v$ detects a disappearance (line 5).

If the message contains two segments, i.e., $m s g(v) \cdot p_{2} \neq \emptyset$ and $m s g(v) \cdot p_{3}=\emptyset$, then node $v$ would detect an expansion or contraction (lines 6-8). As illustrated in Figure 5(f) and (h), the first segment of expansion is on inserted edges (line 7 ), while the first segment of contraction is on deleted edges (line 8).

There are four types of changes, i.e., merge, split, self-merge, and partial-split that have four traversal segments (lines 9-15). In the cases of merge and selfmerge, the first segment is on inserted edges (lines 10-12). Merge and self-merge can then be distinguished by the second and fourth segments of traversals. The second and fourth segments have different areal object identities for a merge, and have the same areal object identity for a self-merge. While for split and partial-split, the first segment is on deleted edges (lines 13-15). The first and third segments have the same areal object identity for a split, and have different areal object identities for a partial-split.

\section{Evaluation}

The decentralized algorithm in Section 5 was evaluated in a simulation environment. Repast (http://repast.sourceforge.net/) was used for simulation. The 
directed planar graph structure was built by Delaunay triangulation in Repast. The planar graph consists of 500 sensor nodes that are deployed in a region of $800 \times 800$ square units, with a communication range of 80 units.

In the experiment, we had about 2000 simulation runs, and each type of change had about 250 runs. An areal object is simulated as one or several $r \times r$ square boxes, as examples in Figure 8. From the 1st to the 2000th simulation runs, the parameter $r$ gradually increase, and thus the number of active nodes should also gradually increase. At each simulation run, a specific type of spatial change can occur at randomized locations within the sensor network. Figure 8 (a) and (b) show examples of merge and partial-split that had occured at different locations of the sensor network.

Our algorithms were installed in each sensor node in the network. In all the simulation runs, the eight different types of changes were correctly detected by the directed planar graph structure, as long as the granularity of observation is set up appropriately based on the granularity of areal objects.

The experiment investigated the scalability of the algorithm. The scalability was measured by number of messages sent with increasing number of active nodes. The results of power regression analysis for appearance, expansion, and merge, for example, is shown in Figure 9(a), (b), and (c) respectively. Figure 9(d) lists the results of regression analysis for the eight types of changes. A power regression $\left(y=a \times x^{b}\right)$ is used to fit the 250 plotted results for each type of change. All the regression results achieve high goodness of fit, with the range of $R^{2}$ from 0.9551 to 0.993 . As in Figure 9(d), there is $b<1$ for all the eight types of changes, and thus our algorithm should have an order of $O(n)$ or less. The experiment shows that our decentralized algorithm is highly scalable.

To compare with our decentralized algorithm, we also implement a centralized sense-and-transmit algorithm, in which each active node will simply forward its sensor reading to a sink by multi-hop routings. The results of the centralized algorithm is shown in the last row of Figure 9(d). By comparing $a$ and $b$ in $y=a \times x^{b}$, it is clear that our decentralized algorithm is more message efficient and scalable than a centralized algorithm.
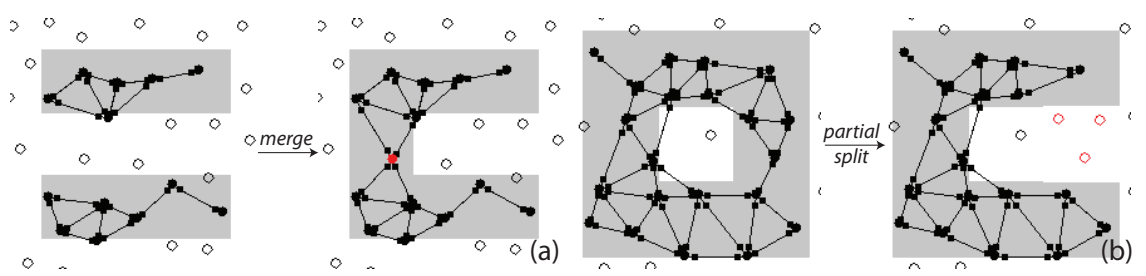

Fig. 8. Spatial changes occur at randomized locations within a sensor network. 


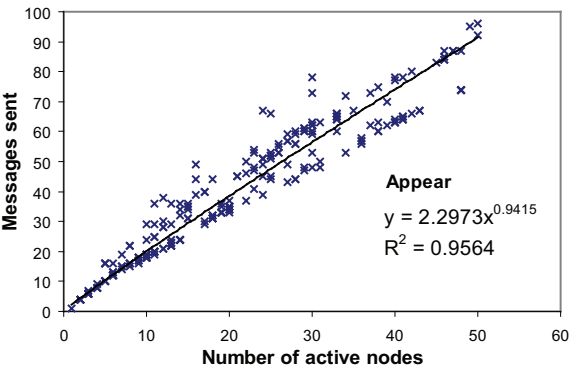

(a) appearance

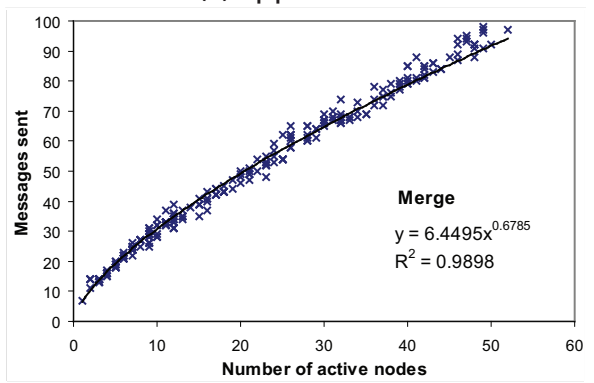

(c) merge

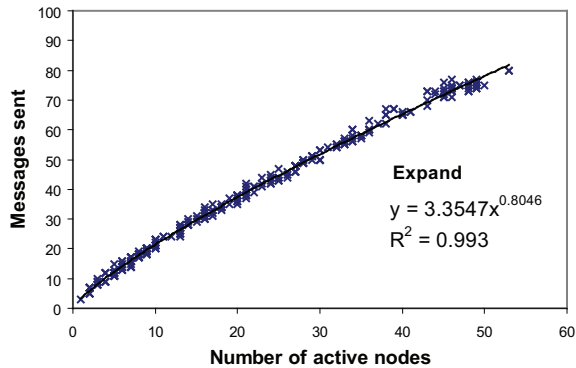

(b) expansion

\begin{tabular}{l|l|l|l} 
Types of change & $a$ & $b$ & $R^{2}$ \\
\hline Appearance & 2.2973 & 0.9415 & 0.9564 \\
Disappearance & 2.3347 & 0.9353 & 0.9551 \\
Expansion & 3.3547 & 0.8046 & 0.9930 \\
Contraction & 3.4046 & 0.8014 & 0.9926 \\
Merge & 6.4495 & 0.6785 & 0.9898 \\
Split & 7.0449 & 0.6552 & 0.9877 \\
Self-merge & 6.4553 & 0.6934 & 0.9893 \\
Partial-split & 6.2780 & 0.6975 & 0.9896 \\
Centralized & 11.6970 & 1.1079 & 0.9984
\end{tabular}

(d) regression analysis $y=a \cdot x^{b}$

Fig. 9. Scalability of the algorithm.

\section{Conclusion}

In this paper, we have developed a new spatiotemporal data model that enables the detection of qualitative spatial changes in snapshot sequences. In our model, boundaries of areal objects are closed traversable trails. Eight types of changes of areal objects can also be represented and distinguished by eight different closed trails that consist of inserted and/or deleted edges. We have also developed a decentralized algorithm for WSNs to detect changes by in-network aggregations. The experiment proves that our algorithm is scalable and efficient, and is able to detect changes with different numbers of active nodes. In the future work, our data model can be further developed to support decentralized spatial queries, for example, the query of the topological relations between two spatial regions.

\section{References}

1. M. Duckham, S. Nittel, and M.F. Worboys. Monitoring dynamic spatial fields using responsive geosensor networks. In Proceedings of 13th Annual ACM International Workshop on Geographic Information Systems (GIS05), pages 51-60, 2005.

2. A. Galton. Qualitative Spatial Change. Oxford University Press, 2000.

3. A. Galton. Fields and objects in space, time, and space-time. Spatial Cognition and Computation, 4(1):39-67, 2004. 
4. P. Grenon and B. Smith. SNAP and SPAN: Towards dynamic spatial ontology. Spatial Cognition and Computation, 4(1):69-104, 2004.

5. K. Hornsby and M. Egenhofer. Qualitative Representation of Change. In S. Hirtle and A.U. Frank, eds.: COSIT 1997, Volume 1329 of Lecture Notes in Computer Science, pages 15-33. Springer, Heidelberg, 1997.

6. J. Lian, L. Chen, K. Naik, Y. Liu, and G.B. Agnew: Gradient boundary detection for time series snapshot construction in sensor networks. IEEE Transactions on Parallel and Distributed Systems, 18(10):1462-1475, 2007.

7. J. Jiang and M. Worboys. Detecting basic topological changes in sensor networks by local aggregation. ACM GIS, Irvine, CA, USA, 2008.

8. J. Jiang and M. Worboys. Event-based topology for dynamic planar areal objects. International Journal of Geographical Information Science, 23(1):33-60, 2009.

9. I. Mau, K. Hornsby, and I.D. Bishop. Modeling geospatial events and impacts through qualitative change. In T. Barkowsky et al., eds.: Spatial Cognition V, Volume 4387 of Lecture Notes in Artificial Intelligence, page 156-174. Springer, Berlin, 2007.

10. D.J. Peuquet and N. Duan. An event-based spatiotemporal data model (ESTDM) for temporal analysis of geographical data. International Journal of Geographical Information Systems, 9:7-24, 1995.

11. D.J. Peuquet. Making Space for Time: Issues in Space-Time Data Representation. Geoinformatica. 5(1):11-32, 2001.

12. F.P. Preparata and M.I. Shamos. Computational Geometry: An Introduction. Springer, New York, 1985.

13. M.J. Sadeq and M. Duckham. Effect of neighborhood on in-network processing in sensor networks. In T.J. Cova et al., eds.: GIScience 2008, LNCS, volume 5266, pages 133-150. Springer, Heidelberg, 2008.

14. C.E. Shannon. Communication in the presence of noise. In Proceedings of the Institute of Radio Engineers 37(1), pages 10-21, 1949.

15. P. Skraba, Q. Fang, A. Nguyen, and L. Guibas. Sweeps over wireless sensor networks. In IPSN 06: Proceedings of the 5th International Conference on Information Processing in Sensor Networks. pages 143151, 2006.

16. T. Wark, P. Corke, P. Sikka, L. Klingbeil, Y. Guo, C. Crossman, P. Valencia, D. Swain, and G. Bishop-Hurley. Transforming Agriculture through Pervasive Wireless Sensor Networks. IEEE Pervasive Computing, 6:2(50-57), 2007

17. M. Worboys. Event-oriented approaches to geographic phenomena. International Journal of Geographical Information Science, 19(1):128, 2005.

18. M. Worboys and M. Duckham. Monitoring qualitative spatiotemporal change for geosensor networks. International Journal of Geographical Information Science, 20(10):1087-1108, 2006. 